\title{
Una aproximación para una propuesta metodológica híbrida entre lo cuantitativo y lo cualitativo para el estudio de las retransmisiones deportivas en la radio española
}

\section{An approach to a hybrid methodological proposal between quantitative and the qualitative techniques for the study of the sport broadcastings in the Spanish radio}

\author{
Francisco Javier Herrero Gutiérrez \\ Universidad de Salamanca
}

\section{Resumen}

Actualmente, en España, las audiencias de radio de las retransmisiones deportivas están aumentando vertiginosamente. Sirva como dato que, según los números referentes a la primera oleada de 2008 del Estudio General de Medios (EGM), el Carrusel Deportivo de la cadena SER -programa del que se han obtenido los fragmentos sobre los que se ejemplifica este artículo- es el tercer programa del ranking de los programas más escuchados de la radio española. A partir de aquí, este artículo pretende ser un punto de partida para analizar si existe o no una hipotética pobreza de recursos lingüísticos en dicho tipo de programas: las retransmisiones radiofónicas deportivas. Éste sería el primero de los dos paradigmas que se presentan. Sólo corroborando esta hipótesis de partida, se podría subir un escalón más y tratar de sacar conclusiones del porqué de eso. Pero, obviamente, para subir al segundo peldaño, antes hay que construir el primero. ¿Y cómo hacerlo? Dada la complejidad que ello conlleva, con este artículo, a través de un pequeño estudio-ejemplo muy concreto, basado en tres fragmentos radiofónicos de programas deportivos, se pretende defender la idea de que las investigaciones que traten este tema no deben encerrarse en un ámbito concreto de la investigación, sino que lo que se ha de buscar es un híbrido metodológico que combine tanto técnicas cuantitativas como cualitativas -estas últimas, basadas principalmente en las estudios de Teun A. Van Dijk y su análisis del discurso- englobándolas bajo un doble nivel de estudio: textual y contextual.

Palabras clave: radio, retransmisiones deportivas, lenguaje deportivo, recursos lingüísticos.

\begin{abstract}
Nowadays, in Spain, the radio hearings of the sport broadcastings are vertiginously increasing. According to the referring numbers to the first big wave of the General Means Study (EGM) in 2008, the program "Carrusel Deportivo" of the channel "Cadena SER" - program from where the fragments on which this article is exemplified have been obtained -is the third program in the ranking of the most listened radio programs in Spain. From this fact, this article pretends to be a departure point in order to analyze if there is or not a hypothetical poverty of linguistic resources in that type of programmes: the radio sport broadcastings. This one would be the first of paradigms that appears. Only confirming this departure hypothesis would be possible to raise one more step and try to get conclusions about that. But, obviously, to raise a second step, it is necessary to have the first one built. And how can we do this? Since it entails a great complexity, this article, through a small and very concrete study-example, based on three fragments of sport radio programmes, tries to defend the idea that the investigations about this subject do not have to be locked in a concrete scope, but we need to look for a technical methodological hybrid that combines quantitative and qualitative techniques -these last ones based on the studies of Teun A. Dijk and his analysis about the speech-including them into a double level of study: textual and contextual.
\end{abstract}

Key words:radio, sport broadcastings, sport language, linguistic resources. 
Herrero, F. J. (2009). Una aproximación para una propuesta metodológica híbrida entre lo cuantitativo y lo cualitativo para el estudio de las retransmisiones deportivas en la radio española.

Revista Internacional de Ciencias del Deporte. 15(5), 46-63. http://www.cafyd.com/REVISTA/01504.pdf

\section{Introducción}

$\mathrm{E}$ n España se han ido sucediendo diversos acontecimientos clave que han venido marcando el devenir de la relación entre el deporte -definido por Santiago Coca, como "una forma de entender la vida” (Solar, 2007, 11) - y la comunicación, tales como el Mundial de fútbol de España de 1982 o los Juegos Olímpicos de Barcelona diez años después. Puntos de inflexión que lo que han hecho es corroborar que "con el paso del tiempo, el clamor por los acontecimientos deportivos, el interés de los políticos para hacerse con su control, la mayor demanda de información sobre el deporte, el paso de juego a deporte espectáculo y la entrada en la materia deportiva de las ciencias y la economía fue motivación para que los responsables de los medios cambiasen su mentalidad y comenzasen a considerar que ese género específico del periodismo no era la cenicienta de la información, sino que por el atractivo que tenía entre los ciudadanos se había colocado en paridad con los otros géneros específicos” (Alcoba, 2005, 66).

Así, poco a poco, el deporte ha ido adquiriendo una mayor importancia, que merece un estudio aparte, de ámbito internacional. Pero este pequeño artículo, lejos de ello, pretende acotarse a un nivel nacional, en España. Antes de la aparición de la televisión, la radio - "un medio vivo que permite el comentario rápido" (Hernando Cuadrado y Hernando García-Cervigón, 2006, 25)- ha venido siendo el transmisor de este tipo de acontecimientos deportivos. Tras la aparición de la propia televisión, lejos de desbancar a la radio, ésta ha adquirido mayor relevancia en la tesitura comunicacional. Quepa aquí mencionar un párrafo de una de las obras textuales de Antonio Alcoba: "La perfección tecnológica de las comunicaciones alcanzó a los medios de comunicación y la radio se interesa por el deporte, sobre todo una vez que son posibles las retransmisiones en directo. El 2 de julio de 1921, la KDKA, una de las primeras compañías radiofónicas norteamericanas consiguió retransmitir, de forma clandestina y en directo, el último asalto de boxeo entre Dempsey y Carpentier, consiguiendo que más de 200.000 personas tuvieran información al momento del mismo. Aquel éxito pone en marcha todo un movimiento periodístico radiofónico culminado, en 1926, con la retransmisión del combate entre Dempsey y Tunney, escuchado por quince millones de personas” (Alcoba, 1993, 65).

\section{La radio deportiva en España}

El boom norteamericano mencionado en la introducción se fue extrapolando mundialmente y, a día de hoy, debido a las costumbres que existen en España, las diferentes emisoras radiofónicas dedican gran parte de su programación a emitir contenidos deportivos. Sin ir más lejos, las principales emisoras, y las más escuchadas, ofrecen a sus oyentes durante los siete días de la semana, programas deportivos a nivel nacional y programas locales deportivos en diferentes franjas horarias, según la emisora.

Pero el tema de los programas con contenidos deportivos nacionales va mucho más allá durante los fines de semana. Son los sábados y los domingos cuando las diferentes emisoras radiofónicas incluyen en sus parrillas multitud de horas dedicadas a la información deportiva. Y muchos son los receptores de este tipo de programas. 
Herrero, F. J. (2009). Una aproximación para una propuesta metodológica híbrida entre lo cuantitativo y lo cualitativo para el estudio de las retransmisiones deportivas en la radio española.

Además, en este sentido, el de la recepción, podemos ver cómo el sonido radiofónico influye no sólo en el ser activo, aquél que enciende la radio para escucharla por hobby, sino también en el ser pasivo: aquél que aunque no le guste ese tipo de programas lo está escuchando, o al menos oyendo ${ }^{1}$, porque no le queda otro remedio pero que, poco a poco, se ha ido aficionando: "La realidad indica que radio y televisión han creado nuevos aficionados al deporte, de manera importantísima en el sector femenino, otrora remiso ante la cuestión deportiva” (Alcoba, 1993, 68).

Para poner de manifiesto la última idea señalada, a continuación presento los diferentes programas con contenido deportivo de las cinco principales emisoras de radio en España $^{2}$ (Tabla 1). Hay que tener en cuenta que las siguientes programaciones suelen tener vigencia, aproximadamente, de septiembre a junio.

Tabla 1. Espacios deportivos programados por las cinco principales emisoras españolas de radio para el periodo comprendido entre septiembre 2007 - junio 2008

\begin{tabular}{|c|c|c|c|}
\hline & LUNES - VIERNES & SÁBADO & DOMINGOS \\
\hline CADENA SER ${ }^{3}$ & 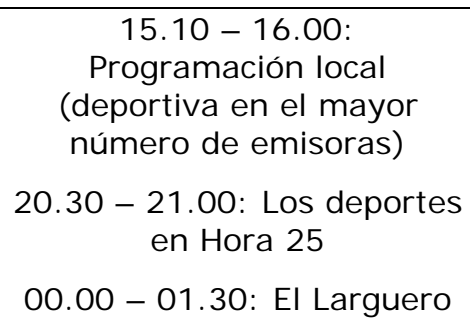 & $\begin{array}{c}18.00 \text { - 00.00: } \\
\text { Carrusel Deportivo } \\
00.00 \text { - 01.00: El } \\
\text { Larguero }\end{array}$ & $\begin{array}{c}\text { 12.00 - 14.00: Programación } \\
\text { local (deportiva en el mayor } \\
\text { número de emisoras) } \\
\text { 15.30 - 23.00: Carrusel } \\
\text { Deportivo } \\
23.30 \text { - } 01.00: \text { El Larguero }\end{array}$ \\
\hline CADENA COPE $^{4}$ & $\begin{array}{l}14.45 \text { - 15.00: Los deportes } \\
\text { en La Palestra } \\
00.00 \text { - 01.30: El Tirachinas }\end{array}$ & $\begin{array}{l}\text { 19.00 - 00.00: } \\
\text { Tiempo de J uego } \\
\text { 00.00 - 01.00: El } \\
\text { Tirachinas }\end{array}$ & $\begin{array}{c}16.00 \text { - 23.00: Tiempo de } \\
\text { Juego } \\
\text { 23.30 - 01.00: El Tirachinas }\end{array}$ \\
\hline $\begin{array}{l}\text { RADIO } \\
\text { NACIONAL DE } \\
\text { ESPAÑA - } \\
{\text { RADIO } 1^{5}}\end{array}$ & $\begin{array}{c}20.30 \text { - 21.00: Radiogaceta } \\
\text { de los deportes }\end{array}$ & $\begin{array}{c}14.30 \text { - 15.00: } \\
\text { Avance Deportivo } \\
\text { 18.00 - 00.00: } \\
\text { Tablero Deportivo }\end{array}$ & $\begin{array}{c}14.30 \text { - 15.00: Avance } \\
\text { Deportivo } \\
\text { 16.00 - 00.00: Tablero } \\
\text { Deportivo }\end{array}$ \\
\hline ONDA CERO $^{6}$ & $\begin{array}{c}15.00 \text { - 16.00: Onda } \\
\text { Deportiva (programación } \\
\text { local) } \\
21.05 \text { - 21.20: Los deportes } \\
\text { en La Brújula } \\
00.00 \text { - 01.30: Al Primer } \\
\text { Toque }\end{array}$ & $\begin{array}{l}\text { 18.00 - 01.00: } \\
\text { Radioestadio }\end{array}$ & 16.00 - 01.00: Radioestadio \\
\hline PUNTO RADIO $^{7}$ & $\begin{array}{l}15.00 \text { - 16.00: Juego Limpio } \\
\text { (Prog. local) } \\
20.30 \text { - 21.00: Deportes en } \\
\text { De Costa a Costa } \\
00.00 \text { - 01.30: El Mirador }\end{array}$ & $\begin{array}{l}\text { 14.30 - 15.00: } \\
\text { Juego Limpio } \\
\text { 18.00 - 01.00: El } \\
\text { Mirador de Liga }\end{array}$ & $\begin{array}{c}14.30 \text { - 15.00: Juego Limpio } \\
16.00 \text { - 23.00: El Mirador de } \\
\text { Liga } \\
23.30 \text { - 01.30: El Mirador }\end{array}$ \\
\hline
\end{tabular}


Herrero, F. J. (2009). Una aproximación para una propuesta metodológica híbrida entre lo cuantitativo y lo cualitativo para el estudio de las retransmisiones deportivas en la radio española.

Revista Internacional de Ciencias del Deporte. 15(5), 46-63. http://www.cafyd.com/REVISTA/01504.pdf

Visto el planteamiento inicial, cabe destacar que a día de hoy, las retransmisiones radiofónicas a las que se les dedica una mayor cobertura radiofónica en España son el fútbol (Primera División de la Liga española de fútbol, Copa del Rey, Champions League, Copa de la UEFA; retransmisiones de finales de las diferentes competiciones europeas aun cuando no hay representación española; Europeos y Mundiales de fútbol); y otras disciplinas deportivas (Europeos y Mundiales de los diferentes deportes y Juegos Olímpicos), estas últimas, con programaciones radiofónicas especiales.

No obstante, de estas retransmisiones mencionadas, hay emisoras que ofrecen una cobertura especial a otros eventos en momentos determinados. Sirva como ejemplo las retransmisiones que viene realizando la Cadena SER de la Superbowl desde hace varios años o las retransmisiones de la Fórmula 1 que la misma emisora realiza desde la temporada 2007-08.

\section{Marco teórico}

Es por todo lo expuesto hasta ahora por lo que existen investigadores que se plantean estudios sobre el uso que del lenguaje se hace en este tipo de retransmisiones radiofónicas deportivas. Los hay, por lo tanto, que hacen una referencia a la fusión entre fútbol y educación: "Fútbol, alineación, poder, dinero pero también comunicación, comprensión, relación social. Fútbol, sobre todo, una necesidad profundamente humana, ante una educación para el trabajo. Fútbol: una oportunidad educativa para el tercer milenio" (Solar, 2007, 136). Estaríamos, pues, ante un análisis del discurso radiofónico deportivo, donde "el énfasis en la naturaleza interactiva y práctica del discurso está naturalmente asociado con un enfoque del uso del lenguaje como interacción oral" (Dijk, 2000b, 23). En este sentido, habríamos de tener en cuenta que la noción de habla estaría relacionada con la espontaneidad, que contiene muchas "propiedades del habla improvisada: pausas, errores, reparaciones, falsos comienzos, repeticiones, superposiciones, etc.” (Dijk, 2000b, 23-24).

A tenor de la idea que ofrece Leopoldo Seijas Candelas, la preocupación radica más bien en el ámbito del purista, defensor a ultranza del idioma: "Nos encontramos, por lo tanto, con que los destinatarios constituyen un público multiforme que impone al lenguaje periodístico ciertos condicionamientos entre los que destaca su llaneza de estilo, es decir, una cierta impregnación del lenguaje oral e incluso coloquial que inquieta a no pocos intelectuales y pone en guardia a los puristas del idioma. No se alarman, sin embargo, los filólogos que consideran el lenguaje como algo vivo y, por lo tanto, sujeto a evolución, y que ha de adaptarse a la corriente variable del tiempo, si no quiere perder el dinamismo que corresponde a su función que, desde nuestro punto de vista, es la comunicativa, más que la estética” (Seijas, 2003, 120).

En contraposición a este punto de vista, podemos también tomar como válida la opinión de Federico Medina, quien acerca de la narración radiofónica de las retransmisiones deportivas, comenta que "no es sólo un discurso informativo que facilita el intercambio directo de sentimientos, ideas y conocimientos, cumple además una finalidad imaginativa. El locutor logra una comunicación poética aprovechando, las posibilidades pictóricas y el poder de sugerencia de algunas imágenes simbólicas y complementa su propuesta estética con las posibilidades sonoras y de ritmo de lengua” (Medina, 1995, 78-79). 
Herrero, F. J. (2009). Una aproximación para una propuesta metodológica híbrida entre lo cuantitativo y lo cualitativo para el estudio de las retransmisiones deportivas en la radio española.

Revista Internacional de Ciencias del Deporte. 15(5), 46-63. http://www.cafyd.com/REVISTA/01504.pdf

Una vez llegados a este punto, sería apropiado mencionar dos grandes paradigmas que se nos plantean. El primero de ellos es tener conocimiento de si realmente existe o no esa llaneza de estilo e impregnación del lenguaje oral y coloquial que plantea Seijas Candelas. Y, en segundo lugar, y en el momento en el que se demuestre esto de forma fundamentada y argumentada, habríamos de subir un segundo escalón y preguntarnos el porqué de ello.

En cualquier caso, antes de aventurarnos a analizar el por qué existe una relativa pobreza del lenguaje, deberíamos corroborar si realmente existe esa limitación intelectual, indagar en ello, ya que "el conocimiento del lenguaje deportivo y su modo de empleo es una asignatura fundamental para cualquier periodista deportivo" (Alcoba, 2005, 114).

A pesar de esta idea que nos plantea Antonio Alcoba, el propio autor del texto ya aventura la posible solución: "Para escribir y hablar de la actividad deportiva nadie va a embarcarse en una complicación adicional para expresar términos y criterios. Hemos querido exponer esta opinión científica con el propósito de hacer ver cómo el deporte ha llegado a interesar en los ambientes académicos, aunque luego todo ese cúmulo de teorías no conduzca a nada efectivo para la marcha y el funcionamiento del lenguaje deportivo” (Alcoba, 2005, 114).

De este análisis micro también podemos obtener soluciones por anticipado desde un nivel macroestructural, como la que ofrece Xoxé Ramón Pousa en el prólogo del libro "Producción Radiofónica”: "La radio es el medio de comunicación que menos literatura genera [...] La radio ha sido una especie de paciente pobre. [...] Sin embargo, pese a su permanente mala salud de hierro, la radio ha conquistado la credibilidad del público, despierta el interés de la audiencia y se ha revelado como el medio de masas más ágil para hacer frente tanto a las grandes crisis de Estado como a los pequeños problemas locales”. (Rodero, 2005, 9).

Dada la idea primera, la de Antonio Alcoba, se da por entendido que el periodista deportivo no está embaucado en esa complicación adicional. ¿Pero realmente no lo está? Probablemente no pero creo que sería necesario hacer un estudio más pormenorizado del tema.

Pero esta misma idea no queda sólo en el lenguaje que emplea el periodista deportivo sino que se puede extender a la totalidad del periodismo, tal y como señala algún autor: "El uso correcto de la lengua en los medios de comunicación lleva años siendo objeto de debate y polémica. El deber o no de conservarla en toda su pureza, y la necesidad de mantenerla incólume, ha enfrentado a los más puristas, defensores del respeto a una norma común que garantiza la comunicación, con aquéllos que sienten la necesidad de permitir que la lengua se desarrolle como algo vivo y cambiante, reflejo de los procesos que también experimenta el hombre. En su uso habitual, orientado hacia la comunicación con nuestros semejantes, el lenguaje se limita a facilitar la comprensión del interlocutor. Por eso la lengua se va cargando de errores y solecismos que, a la vez que la hacen evolucionar, la degradan. Ante esta situación, son muchos, y desde hace mucho tiempo, los que han reivindicado la necesidad de la aplicación de una norma estricta, que evite la quizá inevitable degeneración del idioma”. (Escribano, 2006, 1516). Estaríamos pues ante un análisis social del discurso, que "se ocupa precisamente de 
Herrero, F. J. (2009). Una aproximación para una propuesta metodológica híbrida entre lo cuantitativo y lo cualitativo para el estudio de las retransmisiones deportivas en la radio española.

Revista Internacional de Ciencias del Deporte. 15(5), 46-63. http://www.cafyd.com/REVISTA/01504.pdf

las interrelaciones entre las propiedades locales y globales del texto y el habla social" (Dijk, 2000b, 26).

También, todo un lenguaje periodístico está bajo un envoltorio social por lo que se debe tener en cuenta la relación entre la sociedad y la comunicación: "No es de extrañar que el cambio social acompañe a la planificación formal de la lengua, pues la planificación formal, relacionada con la gestión del cambio, es en sí misma un ejemplo de cambio social. En un mundo estable, de completo equilibrio, en el cual cada día se parece al día anterior y al día siguiente, y en el cual todos los integrantes de la sociedad están satisfechos con esa situación, es poco probable que surja la planificación lingüística” (Cooper, 1997, 194).

En este artículo se recoge lo que podría ser un grano de arena en el desierto y pretende animar a otros investigadores e interesados en la materia a tratar de corroborar el primero de los paradigmas presentados anteriormente.

\section{Material}

A la hora de escoger el siguiente material se ha tenido en cuenta que, en España, hay un deporte por encima de todos. Es, sin duda, el deporte rey. El fútbol es una "religión laica” (Vázquez, 2003, 160) e, incluso, va mucho más allá: "Representa el triunfo absoluto del imperio del ocio sobre la dictadura del trabajo. La libertad del sentimiento impuesta a la sumisión laboral: el fútbol tras cien años de rodaje se ha adelanto a los tiempos para hacernos evidente que nuestra educación olvida lo transcendente en función de lo pragmático” (Solar, 2007, 134). El propio Solar Cubillas afirma que el fútbol "ha logrado imponerse en la 'civilización del ocio'. Su victoria, por lo universal y lo contundente constituye una denuncia o, al menos una advertencia al proceso educativo generalizado a lo largo del siglo” (Solar, 2007, 134).

Por ello, una retransmisión futbolística parece adecuada para tomar varios fragmentos a modo de ejemplos. En cuanto al medio escogido, éstos han sido seleccionados de la cadena SER ya que, en lo que a las retransmisiones deportivas se refiere, es la emisora más sintonizada según los datos que arroja periódicamente el EGM.

Entrando en materia, concretamente se han escogido tres fragmentos. Así, por una parte, el 18 de mayo de 2008 finalizaba el campeonato liguero de la Primera División de la Liga española. Ese domingo, cuatro equipos se jugaban un puesto de descenso: Osasuna, Real Zaragoza, Real Valladolid y Recreativo de Huelva.

Los partidos que afectaban a dichas escuadras eran tres y se jugaron todos a la misma hora: Racing de Santander - Osasuna, Real Mallorca - Real Zaragoza, y Recreativo de Huelva - Real Valladolid.

Los diversos partidos fueron retransmitidos por diversas emisoras radiofónicas; entre ellas, por la cadena SER, dentro de su programa Carrusel Deportivo ${ }^{8}$.

En este artículo se pretende analizar una porción de la comunicación oral de esta retransmisión en concreto, tomando como material radiofónico al mencionado programa deportivo y, dentro de éste, los nueve minutos y 34 segundos que abarcan desde que el Racing de Santander marca su primer y único gol hasta que finaliza el partido entre el Real Mallorca y Real Zaragoza, último en concluir de entre los tres choques reseñados. 
Herrero, F. J. (2009). Una aproximación para una propuesta metodológica híbrida entre lo cuantitativo y lo cualitativo para el estudio de las retransmisiones deportivas en la radio española.

Un mes después, se disputaba la Eurocopa de Fútbol. Varios momentos clave pero dos por encima del resto. La tanda de penaltis entre Italia y España el 22 de junio de 2008 a las 11 y cuarto de la noche (partido de cuartos de final), que a la postre, sería el fragmento televisivo más visto 9 ; y por otra parte, la final que disputaron las selecciones española y alemana el 29 de junio de 2008.

De estos dos partidos, se ha analizado, por una parte, los ocho minutos y 45 segundos (aproximados) que duró la tanda de penaltis del partido Italia - España; por otra parte, un minuto y 35 segundos que giran en torno al gol de Fernando Torres; tanto que, a la postre, serviría para que España se proclamase campeona de Europa. También, al igual que el anterior, estos dos fragmentos han sido extraídos de las retransmisiones realizadas por la cadena SER.

Por lo tanto, en total se ha realizado un análisis de tres fragmentos diferentes, que pueden ser considerados como tres de los fragmentos futbolísticos que atañen al fútbol español que han sido retransmitidos con una mayor pasión ${ }^{10}$.

\section{Método y Resultados}

Durante los minutos analizados del primer fragmento (retransmisión del 18 de mayo de 2008), intervienen durante la retransmisión un total de nueve personas: tres desde un estudio, dos desde Mallorca, dos desde Santander y dos desde Huelva.

Transcrito ese texto, y realizando un desglose gramatical, a un nivel sintácticomorfológico tenemos los siguientes resultados (Tabla 2 y Figura 1):

Tabla 2. Desglose gramatical, en número de veces y porcentaje, del fragmento radiofónico analizado correspondiente al 18 de mayo de 2008

\begin{tabular}{|c|c|c|}
\hline Tipología & Número & $\%$ \\
\hline $\begin{array}{c}\text { Nombres propios } \\
\text { (Jugadores, entrenadores, } \\
\text { periodistas, comentaristas, } \\
\text { ciudades y continentes) }\end{array}$ & 164 & 9,2 \\
\hline $\begin{array}{c}\text { Nombres de equipos de } \\
\text { fútbol }\end{array}$ & 95 & 5,3 \\
\hline Término “gol” & 25 & 1,4 \\
\hline $\begin{array}{c}\text { Verbos } \\
\text { Perífrasis verbales }\end{array}$ & 197 & 11 \\
\hline Sustantivos & 265 & 14,8 \\
\hline Adjetivos & 66 & 3,7 \\
\hline Adverbios & 96 & 5,4 \\
\hline $\begin{array}{c}\text { Resto de términos } \\
\text { (Preposiciones, } \\
\text { conjunciones, artículos, } \\
\text { etc.) }\end{array}$ & 818 & 45,8 \\
\hline TOTAL & 1.787 & 100 \\
\hline
\end{tabular}


Herrero, F. J. (2009). Una aproximación para una propuesta metodológica híbrida entre lo cuantitativo y lo cualitativo para el estudio de las retransmisiones deportivas en la radio española.

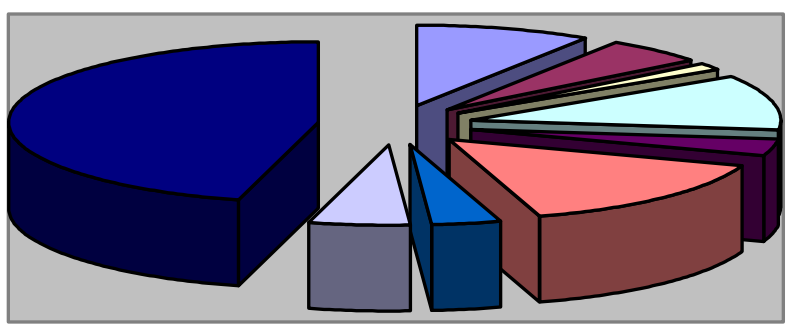

\begin{tabular}{|l|}
\hline Nombres propios \\
$\square$ Nombres de equipos \\
$\square$ Gol \\
$\square$ Verbos \\
$\square$ Perífrasis verbales \\
$\square$ Sustantivos \\
$\square$ Adjetivos \\
$\square$ Adverbios \\
$\square$ Otrostérminos \\
\hline
\end{tabular}

Figura 1. Representación gráfica del desglose gramatical del fragmento radiofónico analizado correspondiente al 18 de mayo de 2008

En cuanto al segundo fragmento (22 de junio de 2008), intervienen cinco personas: dos que están 'in situ' en el campo y tres en el estudio. Según el mismo esquema anterior, los resultados son éstos (Tabla 3 y Figura 2):

Tabla 3. Desglose gramatical, en número de veces y porcentaje, del fragmento radiofónico analizado correspondiente al 22 de junio de 2008

\begin{tabular}{|c|c|c|}
\hline Tipología & Número & $\%$ \\
\hline $\begin{array}{l}\text { Nombres propios (Jugadores, } \\
\text { entrenadores, periodistas, } \\
\text { comentaristas, ciudades y } \\
\text { continentes) }\end{array}$ & 143 & 16,1 \\
\hline Nombres de equipos de fútbol & 1 & 0,1 \\
\hline Término “gol” & 22 & 2,5 \\
\hline Verbos & 162 & 18,3 \\
\hline Perífrasis verbales & 30 & 3,4 \\
\hline Sustantivos & 97 & 11 \\
\hline Adjetivos & 62 & 7 \\
\hline Adverbios & 75 & 8,5 \\
\hline $\begin{array}{l}\text { Resto de términos (Preposiciones, } \\
\text { conjunciones, artículos, etc.) }\end{array}$ & 293 & 33,1 \\
\hline TOTAL & 885 & 100 \\
\hline
\end{tabular}


Herrero, F. J. (2009). Una aproximación para una propuesta metodológica híbrida entre lo cuantitativo y lo cualitativo para el estudio de las retransmisiones deportivas en la radio española.

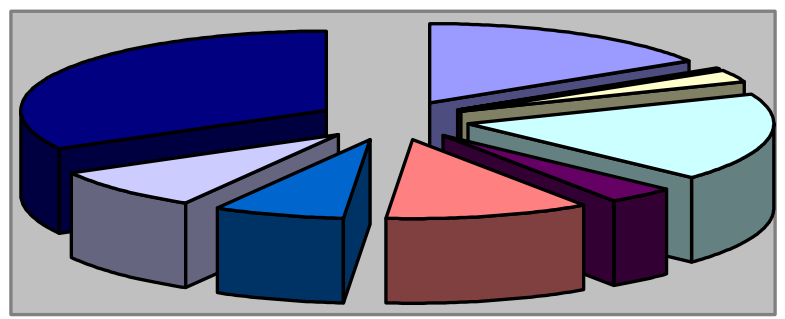

\begin{tabular}{|l}
$\square$ Nombres propios \\
$\square$ Nombres de equipos \\
$\square$ Gol \\
$\square$ Verbos \\
$\square$ Perífrasis verbales \\
$\square$ Sustantivos \\
$\square$ Adjetivos \\
$\square$ Adverbios \\
$\square$ Otrostérminos \\
\hline
\end{tabular}

Figura 2. Representación gráfica del desglose gramatical del fragmento radiofónico analizado correspondiente al 22 de junio de 2008

Por último, en lo que se refiere al tercer fragmento (retransmisión del 29 de junio de 2008), los resultados obtenidos son éstos (Tabla 4 y Figura 3):

Tabla 4. Desglose gramatical, en número de veces y porcentaje, del fragmento radiofónico analizado correspondiente al 29 de junio de 2008

\begin{tabular}{|c|c|c|}
\hline Tipología & Número & $\%$ \\
\hline $\begin{array}{c}\text { Nombres propios (Jugadores, } \\
\text { entrenadores, periodistas, } \\
\text { comentaristas, ciudades y } \\
\text { continentes) }\end{array}$ & 27 & 20,8 \\
\hline $\begin{array}{c}\text { Nombres de equipos de fútbol } \\
\text { Término “gol” }\end{array}$ & 0 & 0 \\
\hline Verbos & 21 & 5,4 \\
\hline Perífrasis verbales & 1 & 16,2 \\
\hline Sustantivos & 11 & 8,5 \\
\hline Adjetivos & 15 & 11,5 \\
\hline Adverbios & 2 & 1,5 \\
\hline Resto de términos & 130 & 35,3 \\
\hline artículos, etc.) & & 100 \\
\hline TOTAL & & \\
\hline (Preposiciones, conjunciones, & & \\
\hline
\end{tabular}


Herrero, F. J. (2009). Una aproximación para una propuesta metodológica híbrida entre lo cuantitativo y lo cualitativo para el estudio de las retransmisiones deportivas en la radio española.

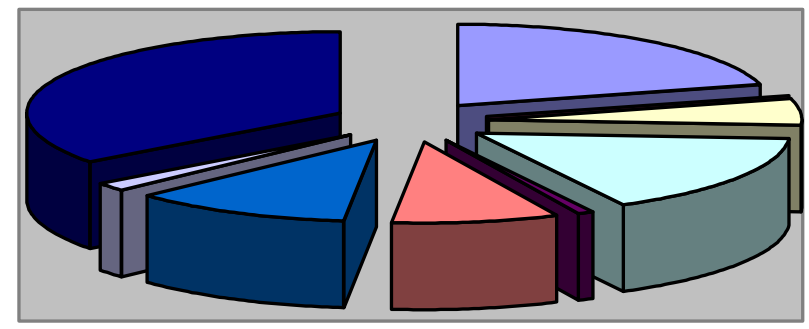

\begin{tabular}{|l|}
\hline Nombres propios \\
$\square$ Nombres de equipos \\
$\square$ Gol \\
$\square$ Verbos \\
$\square$ Perífrasis verbales \\
$\square$ Sustantivos \\
$\square$ Adjetivos \\
$\square$ Adverbios \\
$\square$ Otrostérminos \\
\hline
\end{tabular}

Figura 3. Representación gráfica del desglose gramatical del fragmento radiofónico analizado correspondiente al 29 de junio de 2008

Una vez visto estos resultados y dado el número total de palabras analizadas en el primer fragmento -el más amplio de los tres con un total de 1.787-, obtenemos que poco más del $50 \%$ corresponde a nombres propios, sustantivos, verbos y perífrasis verbales, adjetivos y adverbios. El resto de palabras -conjunciones, preposiciones, artículos, etc.- conforman casi el $46 \%$.

De entre las palabras analizadas, cabe destacar que entre nombres propios y sustantivos se llega al 30,7\%, mientras que el número de verbos más el de perífrasis verbales es del 14,4\%. En lo que se refiere al número de adjetivos, éste es del 3,7\% mientras que el de adverbios es del 5,4\%.

De los otros dos fragmentos, destaca que aumenta el número de nombre propios $(16,1 \%$ y 20,8\% respectivamente) y se anula el de términos en los que se nombra a equipos de fútbol $(0,1 \%$ y $0 \%$, respectivamente), pero hay que recordar que estos dos fragmentos ya no se corresponden a un campeonato liguero sino a un torneo europeo de selecciones. Del segundo fragmento, cabe destacar también que se supera el $20 \%$ en la suma de verbos y perífrasis verbales y se emplean también más adjetivos (7\%) y adverbios $(8,5 \%)$ que en el primero de los fragmentos en este artículo presentados. Por último, cabe mencionar que, de los tres fragmentos analizados, el más rico en adjetivos es el tercero con un $11,5 \%$.

Poniéndonos en un punto extremista, imaginemos que extrapolamos estos datos al total de retransmisiones de todos los partidos de fútbol de las diferentes emisoras radiofónicas. Si así fuera y tuviéramos estos mismos datos de forma general, ¿podríamos hablar de austeridad lingüística?

Sería entonces ésta otra de las misiones subyacentes a añadir a los dos paradigmas ya presentados: el establecer una barrera fronteriza que nos permitiese hablar o no de austeridad lingüística. ¿Dónde estaría la barrera limítrofe entre la austeridad y la no austeridad en lo que al lenguaje deportivo respecta? Dentro de este límite, ¿también hemos de tener en cuenta la invasión de los anglicismos deportivos, una jerga deportiva que "nos invade sin cesar diariamente” (Alonso, 1996, 111)? 
Herrero, F. J. (2009). Una aproximación para una propuesta metodológica híbrida entre lo cuantitativo y lo cualitativo para el estudio de las retransmisiones deportivas en la radio española.

Revista Internacional de Ciencias del Deporte. 15(5), 46-63. http://www.cafyd.com/REVISTA/01504.pdf

Recordemos, en este punto, el primero de los dos paradigmas planteados: ¿Estamos ante un lenguaje deportivo radiofónico acomodado y falto de recursos? En definitiva, muchos son los interrogantes que se nos plantean y creo que, por ahora, pocas las respuestas que tenemos.

Amén de lo presentado, en futuros estudios habría de hacerse especial hincapié en el análisis contextual y no sólo en el textual. En este artículo, con los ejemplos aquí tratados -tan sólo tres- no se puede realizar un estudio contextual desde el momento en el que creo que sería necesaria una muestra mucho más amplia, que nos otorgara una visión más global y que sirviera, a la vez, para poder realizar una comparativa con las retransmisiones de otros países.

\section{Propuesta metodológica}

Para indagar con más profundidad sobre este asunto, y una vez visto este pequeño análisis -cuantificador -, tendríamos que averiguar qué tipo de metodología deberíamos establecer, con el fin de buscar unas soluciones verdaderamente fiables, que nos ayuden a sentar los cimientos sobre los que construir futuras investigaciones.

Una técnica cuantitativa -“caracterizada como típica de las ciencias duras, ya que es rigurosa, sistemática y objetiva, se centra en el análisis de datos numéricos y utiliza para ello procedimientos estadísticos para el contraste de hipótesis” (Igartua, 2006, 92)podría no ser del todo válida desde el momento en que no se sepan manejar las fronteras entre un correcto e incorrecto lenguaje. Con un análisis de contenido -entendido como "un método de estudio y análisis de comunicación de forma sistemática, objetiva y cuantitativa, con la finalidad de medir determinadas variables” (Wimmer y Dominick, 1996, 170), apoyado en el método científico, no creo que se pudieran establecer todos los ítems -y posteriores soluciones, necesarios para tratar de resolver el primero de los paradigmas; ahora bien, sin duda, serviría de mucha ayuda.

No obstante, de esto no podríamos obviar un tipo de análisis desde un punto de vista más bien sociológico, utilizando técnicas cualitativas. Tomando como base ideas de Van Dijk sobre el concepto de noticia, las retransmisiones deportivas se pueden entender como noticia: "El concepto de la noticia en los medios implica los siguientes conceptos: 1. Nueva información sobre sucesos, objetos o personas; 2. Un programa tipo (de televisión o de radio) en el cual se presentan ítems periodísticos; 3. Un ítem o informe periodístico, como por ejemplo un texto o discurso en la radio, en la televisión o en el diario, en el cual se ofrece una nueva información sobre sucesos recientes" (Dijk, 1990, 17). Hay que evitar la confusión de que la noticia es sólo lo que aparece en prensa. Es por ello que el propio Van Dijk realiza la siguiente aclaración en uno de sus libros: "La mayoría de las veces, nos referimos a la noticia en la prensa, es decir, el discurso o los artículos periodísticos publicados cotidianamente en los diarios. Descuidaremos los ítems de la televisión o la radio aun cuando tengamos en cuenta los resultados de las investigaciones sobre estas noticias [...] ¿Y qué ocurre con los informes meteorológicos, las programaciones de la radio y la televisión, las tiras cómicas, los comentarios de libros o las secciones de artes y espectáculos? Algunos de estos ítems también aportan información nueva” (Dijk, 1990, 17). 
Herrero, F. J. (2009). Una aproximación para una propuesta metodológica híbrida entre lo cuantitativo y lo cualitativo para el estudio de las retransmisiones deportivas en la radio española.

Revista Internacional de Ciencias del Deporte. 15(5), 46-63. http://www.cafyd.com/REVISTA/01504.pdf

Es por ello, y siguiendo las ideas planteadas por Van Dijk que podríamos enfocar el estudio de las retransmisiones deportivas, no sólo desde un mero análisis cuantitativo, sino también cualitativo a través de un "análisis del discurso", entendiendo por éste como "un campo de estudio nuevo, interdisciplinario, que ha surgido a partir de algunas otras disciplinas de las humanidades y las ciencias sociales, como la lingüística, los estudios literarios, la antropología, la semiótica, la sociología y la comunicación oral” (Dijk, 1990, 35-36). Dentro al apartado sociológico, podemos hacer referencia a una idea englobada bajo una sociología de la lengua, entendiendo por ésta como que "la planificación lingüística comprende los esfuerzos deliberados por influir en el comportamiento de otras personas respecto de la adquisición, la estructura o la asignación funcional de sus códigos lingüísticos” (Cooper, 1997, 60).

Además, por otra parte, el propio Van Dijk señala, que “en el marco de la ciencia del texto, precisamente, se podría dedicar sistemáticamente una mayor atención a estas formas de uso de la lengua, de manera que también se trataran los artículos periodísticos, los productos de otros medios de comunicación, las conversaciones, las situaciones e instituciones sociales de un determinado idioma o cultura” (Dijk, 1983, 17). Dentro de la metodología que se propone "el mayor esfuerzo de trabajo todavía va dirigido al análisis gramatical y a las teorías gramaticales” (Dijk, 1983, 17).

Con el análisis del discurso, "el principal objetivo consiste en producir descripciones explícitas y sistemáticas de unidades del uso del lenguaje al que hemos denominado discurso. Estas descripciones tienen dos dimensiones principales a las que podemos denominar simplemente textual y contextual. Las dimensiones textuales dan cuenta de las estructuras del discurso en diferentes niveles de descripción. Las dimensiones contextuales relacionan estas descripciones estructuras con diferentes propiedades del contexto, como los procesos cognitivos y las representaciones o factores socioculturales” (Dijk, 1990: 45-46). Señalemos aquí otra de las ideas de Dijk acerca de que "la mente es social, al adquirirse, compartirse, utilizarse y cambiar socialmente. Muchos aspectos de la estructura social presuponen esos conocimientos y creencias compartidas de los miembros. Gran parte de nuestra mente consiste de representaciones social y culturalmente compartidas” (Dijk, 1999, 295).

Estructuralmente, "los sistemas lingüísticos se asemejan a diferentes formas de aplicación pronominales, que pueden ser diferentes para lenguajes diferentes. Pero un aspecto del contexto comunicativo -entendiendo por contexto como "algo que funciona como trasfondo, marco, ambiente, condiciones o consecuencias” (Dijk, 2000b, 32)-, como el grado de formalidad de la situación o la familiaridad de los participantes en la conversación puede determinar si debe elegirse una forma más formal o una más informal” (Dijk, 1990, 45-46).

Más que en ningún caso, el de las retransmisiones radiofónicas del deporte poseen un doble enfoque textual - contextual, dimensiones ambas que se han de abordar. Para el textual, sirva como ejemplo el presentado anteriormente y que lingüísticamente se podría analizar atendiendo al "aspecto verbal de las emisiones [...] La lingüística distingue metafóricamente diversos niveles de esas emisiones, como si se tratara de edificios o construcciones” (Dijk, 2000a, 27). Por otra parte, sí hay que realizar un análisis mucho más exhaustivo del análisis contextual. Por un lado, debemos tener en cuenta los procesos cognitivos de los habitantes españoles y puede acotarse, perfectamente, a la última década. Por otra parte, sería muy importante establecer los 
Herrero, F. J. (2009). Una aproximación para una propuesta metodológica híbrida entre lo cuantitativo y lo cualitativo para el estudio de las retransmisiones deportivas en la radio española.

puntos fuertes socioculturales: "sobre todo durante los últimos años, existe una amplia tendencia a estudiar el uso de la lengua dentro de su contexto psíquico y social” (Dijk, 1983, 17). En este aspecto, habría de tenerse en cuenta la importante función social que tiene el deporte dentro de la población española. Estaríamos aquí dentro de un contexto, entendido éste no sólo como un "mundo-estado posible, sino al menos una secuencia de mundos-estados. Además, estas situaciones no permanecen idénticas en el tiempo, sino que cambian. Por tanto, un contexto es un transcurso de sucesos” (Dijk, 1993, 274).

Periodísticamente hablando, el deporte juega una función más que importante dentro del panorama informativo. Estamos ante un tipo de discurso diferente: "No es un texto que tiene como ambición trascender las culturas y las barreras locales, ni ser universal. En su proceso de producción intervienen la condición sociocultural de cada locutor, su mentalidad, su localización generacional, las tendencias ambientales de la época, el imaginario de la comunidad a la que pertenece, su posición social, su formación académica” (Medina, 1995, 78).

Finalmente, con todo ello estaríamos hablando de un doble análisis textual-contextual, con lo que ello conlleva y sabiendo las diferencias entre ambos conceptos, ya que "una de las cuestiones naturales a plantear en tal marco, sería si la estructura del discurso, al menos desde un punto de vista gramatical, podría ser explicada con referencia a oraciones (simples y compuestas) por un lado, y la estructura de secuencias de actos de habla y de contexto por otro. En otras palabras: tan pronto como llegamos a una explicación pragmática de las estructuras contextuales, tales como conocimiento y creencias, intenciones, acciones, etc. ¿por qué necesitamos todavía un nivel específico discursivo de análisis y no sólo una descripción de oraciones-en-el-contexto? Por ejemplo, para proporcionar la interpretación necesaria relativa de las frases o para la identificación correcta de los individuos respecto a las oraciones previas de la secuencia discursiva, podríamos también interpretar una frase en relación con la frase previamente expresada en el mismo contexto de conversación [...] Aunque no puede negarse que tal aproximación es interesante, y sería ciertamente válida desde el punto de vista del tratamiento cognoscitivo, hay serios argumentos por los que sigue siendo necesario un análisis lingüístico (gramatical) de las secuencias y del discurso incluso dentro de un marco pragmático” (Dijk, 1993, 323).

Por último, a la hora de realizar esta propuesta metodológica, a priori se rechazan otro tipo de análisis de mensajes por diversos motivos. Así, no tenemos en cuenta el análisis retórico porque precisamente lo que se pretende es realizar un análisis del 'qué se dice' y no tanto del 'cómo se dice'. El análisis narrativo no sería usado desde el momento en que no se basa tanto en la improvisación y espontaneidad. Por otra parte, sí se podrían tomar nociones de los análisis semiótico e interpretativo (de hecho, ambas materias ya han sido mencionadas con anterioridad).

\section{Discusión}

En los últimos años, deporte y comunicación han estado estrechamente unidos. Ya en 1995, Federico Medina comentaba: "El aporte de los medios de masas en la difusión del espectáculo futbolístico fue doble: de un lado, contribuyeron a crear el imaginario futbolístico, los conjuntos de percepciones, recuerdos y estados de ánimo asociados al fútbol, a darle forma a la pasión por el juego (a la simpatía o a la indignación); y, por 
Herrero, F. J. (2009). Una aproximación para una propuesta metodológica híbrida entre lo cuantitativo y lo cualitativo para el estudio de las retransmisiones deportivas en la radio española.

Revista Internacional de Ciencias del Deporte. 15(5), 46-63. http://www.cafyd.com/REVISTA/01504.pdf

otro, crearon un discurso, una poética, una retórica y una forma de argumentar y reflexionar sobre el fútbol que se incorporó al uso común de la lengua" (Medina, 1995, 74).

En el caso español, sirva como ejemplo que, según los datos arrojados por el EGM con respecto a la primera oleada de 2008, el Carrusel Deportivo (Cadena SER) de los domingos es el tercer programa más escuchado de la radio española, con más de 2.000.000 de oyentes ${ }^{11}$. El propio Carrusel Deportivo de los sábados es el cuarto programa más escuchado de la radio en España con más de 1.800.000 de oyentes.

En el top-ten también estaría El Larguero, de la cadena SER, si bien este programa, aunque deportivo, se saldría fuera del objeto de estudio. No obstante, los programas deportivos de las diferentes emisoras se encuentran entre los más escuchados por sus oyentes de entre todos los que ofrecen en su parrilla.

De tal forma, debe desarrollarse un sistema en el que englobemos el estudio de las retransmisiones deportivas, tomando, por un lado, técnicas cuantitativas ya conocidas y, por otra parte, basándonos en las ideas de Van Dijk, teniendo en cuenta cinco factores: cuatro de descripción gramatical -fonología, morfología, sintaxis y semántica- y uno pragmático -actos del habla (Dijk, 1990). El propio Dijk lo recoge (Tabla 5) en un cuadro-resumen (Dijk, 1990, 52):

Tabla 5. Niveles y dimensiones estructurales del análisis del discurso y subcategorías

\begin{tabular}{|c|c|c|c|c|c|}
\hline & Micro & Macro & Estilo & Retórica & Superestructura \\
\hline Fonología & $\mathrm{X}$ & & $\mathrm{X}$ & $\mathrm{X}$ & \\
\hline Morfología & $\mathrm{X}$ & & $\mathrm{X}$ & $\mathrm{X}$ & \\
\hline Sintaxis & $\mathrm{X}$ & $(\mathrm{X})$ & $\mathrm{X}$ & $\mathrm{X}$ & \\
\hline Semántica & $\mathrm{X}$ & $\mathrm{X}$ & & $\mathrm{X}$ & $\mathrm{X}$ \\
\hline Pragmática & $\mathrm{X}$ & $\mathrm{X}$ & & $\mathrm{X}$ & $\mathrm{X}$ \\
\hline
\end{tabular}

Desde un punto de vista de análisis del discurso, podríamos analizar de una forma más exhaustiva frases ${ }^{12}$ como "El Racing entra a Europa y no cambia lo de abajo pero, si marca un gol el Zaragoza, ahora el que cae a Segunda sería el Osasuna, aunque de momento es el Zaragoza el equipo que está en zona de descenso. Reacciones. En Mallorca"; "Juanfran ha dado a Jonathan estando en el suelo. Ha sido todo porque Ayoce le ha ido a recriminar a Juanfran esa entrada, que le habían sacado ya tarjeta amarilla; también está Puñal en el suelo doliéndose de algo pero la tangana, todo comenzó con Ayoce recriminándole a Juanfran la entrada”; “es Oliveira quien era agarrado, toca para atrás de cara para Ayala, que sube al ataque. Ayala sube la pelota para Zapater. Cuarenta y seis. Quedan tres tan sólo. La pone, cuidado, para Sergio García. Quiere darse la vuelta, no controló y se cayó. Fernando Navarro la saca para Ibagaza”; "el gol del Recre le salva porque, aunque ahora el Zaragoza empatara en el minuto que queda, no le sirve para nada. El Zaragoza tendría que meter dos goles. O sea, Zaragoza y Osasuna empatados, se salva Osasuna. El gol del Recre de quién fue, que ni te lo pregunté”. 
Herrero, F. J. (2009). Una aproximación para una propuesta metodológica híbrida entre lo cuantitativo y lo cualitativo para el estudio de las retransmisiones deportivas en la radio española.

Revista Internacional de Ciencias del Deporte. 15(5), 46-63. http://www.cafyd.com/REVISTA/01504.pdf

Para llegar a este amplio estudio debemos considerar muchos aspectos; quizás los más importantes, nos los da Van Dijk: "La lingüística general tiene como tarea principal el desarrollo de teorías sobre las gramáticas de las lenguas naturales [...] Una gramática explica sobre todo el sistema de normas que forma la base de la producción y la comprensión de los enunciados de una lengua determinada. La descripción de la estructura de estos enunciados tiene lugar en diferentes niveles [...] Una gramática pretende tanto describir oraciones como secuencias de oraciones, si resultase que entre las oraciones de unos enunciados existieran determinadas relaciones, tal y como por ejemplo existen entre palabras y grupos de palabras dentro de una misma oración [...] Las propias oraciones pueden poseer tales estructuras 'secuenciales', a saber, como oraciones compuestas [...] Las relaciones entre oraciones en oraciones compuestas y secuencias son sobre todo de tipo semántico [...] El significado de una oración aislada se denomina, a grandes rasgos, una proposición; este concepto está tomado de la filosofía y la lógica. Por regla general, la proposición se caracteriza como algo que puede ser 'verdadero' o ‘falso' (en una situación determinada) [...] Estas relaciones pueden fundarse en significados (relaciones intencionales) o en relaciones entre referentes o denotados (relaciones extensionales) [...] Las proposiciones pueden estar en suma ligadas entre ellas de dos en dos” (Dijk, 1983, 31-45). Y a partir de aquí, dar un salto mayor y proceder a realizar un análisis macroestructural tomando como noción las conexiones que "se basan en el texto como un todo o por lo menos en unidades textuales mayores. Llamaremos macroestructuras estas estructuras del texto más bien globales. Por consiguiente, podemos llamar microestructuras las estructuras de oraciones y secuencias de textos para diferenciarlas de aquéllas” (Dijk, 1983, 55).

Precisamente, es éste ahora el tema sobre el que estoy trabajando y al que invito a cualquier investigador interesado en la materia a desarrollar; sólo con un contraste real de resultados, podemos alcanzar una globalización limítrofe de lo que puede ser un apasionante estudio.

\section{Conclusiones}

No cabe duda de que, según las principales fuentes de estudios de audiencia en España, los receptores radiofónicos de las retransmisiones deportivas están aumentando a pasos agigantados.

Algunos expertos hablan de pobreza de recursos lingüísticos, pongamos un nuevo ejemplo: "La transgresión idiomática, con chillido incluido, es de norma en las crónicas deportivas orales” (Lázaro, 1999, 595). En relación a las noticias en general, el propio Lázaro Carreter afirma que "suelen ser bastante deficientes y contienen enormes vicios de tipo léxico los cuales no son privativos de la redacción periodística, sino que de ellos nos nutrimos todos y se observan también en la cátedra, en el púlpito y en los tribunales de justicia” ${ }^{\text {" }}$. No obstante, también se han alzado voces a favor de un tipo de lenguaje más coloquial. Así, sirva la idea aportada por García Calvo: “Así que en la lengua no manda nadie, más que el pueblo, que no es nadie [...] La lengua popular y viva no puede nunca cometer faltas, por la razón perogrullesca de que es ella la que establece, allá en el subconsciente, sus propias leyes” (Hernando, 1990, 29).

Los objetivos de este artículo vienen dados en los dos paradigmas planteados en este artículo, con la misión subyacente también mencionada. Para el primero de los paradigmas, el averiguar si realmente existe pobreza de recursos lingüísticos en las 
Herrero, F. J. (2009). Una aproximación para una propuesta metodológica híbrida entre lo cuantitativo y lo cualitativo para el estudio de las retransmisiones deportivas en la radio española.

Revista Internacional de Ciencias del Deporte. 15(5), 46-63. http://www.cafyd.com/REVISTA/01504.pdf

retransmisiones deportivas de la radio española, se propone una metodología que combine las ya muy conocidas técnicas cuantitativas con otras de tipo principalmente cualitativo, y basadas principalmente en nociones arrojadas por Teun A. van Dijk. La misión subyacente estaría encuadrada aquí: el buscar una barrera limítrofe y poner cifras porcentuales a conceptos que pueden ser un tanto subjetivos.

Sólo corroborando la hipótesis de partida, nada sencilla dicho sea de paso, podríamos ampliar la investigación y marcarnos otro tipo de cotas, bien diferentes como, por ejemplo, en caso de que se corrobore esta hipótesis inicial, el porqué sucede eso segundo de los paradigmas presentados en este artículo-; incluso, ir más lejos y, en el caso de asumir una pobreza lingüística del lenguaje deportivo en la radio española, si tanto emisores como receptores como los grandes sistemas estructurales de la comunicación estarían interesados en la modificación o introducción de cambios sustanciales.

En esta última línea planteada, no debemos pasar por alto, por lo tanto, que una de las posibles causas y motivos de esta presunta dejadez lingüística es que, simplemente, el actual lenguaje de la radio en lo que a las retransmisiones deportivas respecta gusta a unos y otros a pesar de que "no es un texto depurado, el locutor no lo puede corregir, modificar o manipular con posterioridad; ni puede realizar una relectura reflexiva" (Medina, 1995, 78).

\section{Notas}

1 Oír: "percibir con el oído los sonidos"; escuchar: "prestar atención a lo que se oye". Definiciones obtenidas de la vigésima segunda edición del diccionario on-line de la Real Academia Española (http://www.rae.es).

2 Información obtenida de las parrillas que hacen referencia a la temporada 2007-08.

3 Programación obtenida de la parrilla de la cadena SER:

http://www.cadenaser.com/parrilla-programacion/, a fecha 15 de mayo de 2008.

${ }^{4}$ Programación obtenida de la parrilla de la cadena COPE:

http://www.cope.es/programacion, a fecha 15 de mayo de 2008.

5 Programación obtenida de la parrilla de Radio Nacional de España-Radio 1: www.rtve.es/archivos/70-5761 FICHERO/Radio1_PARRILLA_3_SEPTIEMBRE_2007.pdf, a fecha 15 de mayo de 2008.

6 Programación obtenida de la parrilla de Onda Cero: http://www.ondacero.es/ondacero/GestorWeb?id=0\&dest=/web/html/programacion/parrilla.j sp, a fecha 15 de mayo de 2008.

7 Programación obtenida de la parrilla de Punto Radio:

http://www. puntoradio.com/programacion/, a fecha 15 de mayo de 2008.

${ }^{8}$ Destaca la relevancia de este programa ya que, según los datos arrojados por el EGM con respecto a la primera oleada de 2008, el Carrusel Deportivo de los domingos (Cadena SER) es el tercer programa más escuchado de la radio española, con más de 2.000.000 de oyentes. De entre todos los programas deportivos que ofrecen las diversas emisoras radiofónicas españolas, el Carrusel Deportivo de los domingos es el más escuchado. Se pueden consultar los datos de todas las emisoras ofrecidas por el EGM en http://www.aimc.es/.

9 Desde que existen los estudios de audiencia televisiva - año 1992- éste ha sido el espacio más visto de la historia de la televisión en España, con una media de 15.370.000 espectadores y con una cuota de pantalla del 77,5\%. El lanzamiento del último penalti, el de Cesc Fábregas, congregó delante de la pequeña pantalla a 15.922.000 espectadores y una cuota del $79,9 \%$. 
Herrero, F. J. (2009). Una aproximación para una propuesta metodológica híbrida entre lo cuantitativo y lo cualitativo para el estudio de las retransmisiones deportivas en la radio española.

Revista Internacional de Ciencias del Deporte. 15(5), 46-63. http://www.cafyd.com/REVISTA/01504.pdf

10 Aquí viene al caso lo que comenta Solar Cubillas sobre el término 'pasión': “Pero creo que para la mayor parte de los mortales pasión, lo que se dice pasión, el trabajo aporta lo justo. Donde de verdad depositamos nuestra carga afectiva y donde de verdad estamos dispuestos a padecer, es en los verdes campos de ese edén terreno que llamamos fútbol" (Solar, 2007, 134).

11 Según el propio EGM, no ha bajado de los dos millones de oyentes desde el año 2004.

12 Ejemplos obtenidos del fragmento radiofónico analizado.

13 Fragmento de texto obtenido del libro de Asunción Escribano Lengua y Medios de Comunicación, quien a su vez hace referencia a ORTEGA, P.: "Lázaro Carreter: 'Los vicios léxicos invaden los discursos'", en YA, 24-8-1985, p. 17.

\section{Referencias bibliográficas}

Alcoba, A. (2005). Periodismo deportivo. Madrid: Editorial Síntesis

Alcoba, A. (1993). Cómo hacer periodismo deportivo. Madrid: Editorial Paraninfo

Alonso, J. (1996). Anglicismos deportivos. Uso y abuso en la información española. Salamanca: Junta de Castilla y León - Consejería de Educación y Cultura.

Cooper, R. L. (1997). La planificación lingüística y el cambio social. España: Cambridge University Press.

Dijk, T. A. (2000a). El discurso como estructura y proceso. Barcelona: Gedisa Editorial.

Dijk, T. A. (2000b). El discurso como interacción social. Barcelona: Gedisa Editorial.

Dijk, T. A. (1999). Ideología: una aproximación multidisciplinaria. Barcelona: Gedisa Editorial.

Dijk, T. A. (1993). Texto y contexto: Semántica y pragmática del discurso. Salamanca: Cátedra.

Dijk, T. A. (1990). La noticia como discurso: comprensión, estructura y producción de la información. Barcelona: Paidós Comunicación.

Dijk, T. A. (1983). La ciencia del texto. Barcelona: Ediciones Paidós.

Escribano, A. (2006). Lengua y medios de comunicación. Salamanca: Librería Cervantes.

Hernando, L. A. y Hernando, A. (2006). Lengua y comunicación en el discurso periodístico de divulgación científica y tecnológica. Madrid: Editorial Fragua.

Hernando, B. (1990). Lenguaje de la prensa. Madrid: Eudema.

Igartua, J. J. (2006). Métodos cuantitativos de investigación en comunicación. Barcelona: Bosch.

Lázaro, F. (1999). El dardo en la palabra. Barcelona: Galaxia Gutenberg-Círculo de Lectores.

Medina, F. (1995). Los narradores deportivos y sus epopeyas cotidianas. Estudios sobre las culturas contemporáneas, 1 (002), 69-106.

Rodero, E. (2005). Producción radiofónica. Madrid: Ediciones Cátedra.

Seijas, L. (2003). Estructura y fundamentos del periodismo especializado. Madrid: Editorial Universitas. 
Herrero, F. J. (2009). Una aproximación para una propuesta metodológica híbrida entre lo cuantitativo y lo cualitativo para el estudio de las retransmisiones deportivas en la radio española.

Revista Internacional de Ciencias del Deporte. 15(5), 46-63. http://www.cafyd.com/REVISTA/01504.pdf

Solar Cubillas, L. V. (2007). Deporte: El gigante virtual. Centro de Estudios Olímpicos.

Vázquez Montalbán, M. (2003). La aznaridad: Por el imperio hacia Dios o por Dios hacia el imperio. Barcelona: Mondadori.

Wimmer, R. y Dominick, J. (1996). La investigación científica de los medios de comunicación: una introducción a sus métodos. Barcelona: Bosch. 